Commun. math. Phys. 3, 187-193 (1966)

\title{
Condensation of Lattice Gases
}

\author{
J. GINIBRE \\ Laboratoire de Physique Théorique, 91. Orsay - France \\ A. Grossmann and D. Ruelle \\ Institut des Hautes Etudes Scientifiques, 91. Bures-sur-Yvette - France
}

Received May 20, 1966

\begin{abstract}
Techniques due to R. L. Dobrushin and R. Griffiths are combined to prove the existence of a first order phase transition at low temperature for a class of lattice systems with non nearest-neighbour interaction.
\end{abstract}

\section{Introduction}

In recent papers, DoBRushrn [2] and GRIFFITHS [5] have proved that a gas with nearest-neighbour attractive interaction on a cubic lattice in $\nu$ dimensions $(v \geqq 2$ ) undergoes a first order phase transition. DoBRusHrN and GRIFFITHS compute explicitly a region where two phases coexist and the pressure is a constant function of density at constant temperature.

While the result and techniques used are not quite new (see [7], [9], [10]), they are important in giving a simple model for proofs of condensation $^{1}$. In this note we shall combine the techniques of DoBRUSHIN and GrIfFiths (these authors worked independently) to prove the existence of a first order phase transition at low temperature for a class of lattice systems with non nearest-neighbour interaction. Our main result is the theorem of Section 3, which the reader may consult at this point. Section 2 contains preparatory material for the proof of the theorem.

\section{Systems with pair interactions on a lattice}

We collect in this section some definitions and known results.

We consider a $\boldsymbol{v}$-dimensional lattice with vertices $\mathbf{k}=\left(k^{1}, \ldots, k^{v}\right)$ where $k^{1}, \ldots, k^{v}$ are integers. Particles on the lattice are assumed to interact through a pair potential $\Phi$ such that $\Phi(\mathbf{k})=\Phi(-\mathbf{k})$ and

$$
\Phi(0)=+\infty, \sum_{k \neq 0}|\Phi(\mathbf{k})|=D<+\infty .
$$

1 One of us (D. R.) has been informed by V. ARNold and R. BALESCU that further results in this direction have been obtained by SINAI and BEREZIN; on the other hand DoBRushin has extended his results to certain lattice gases with non nearest neighbour interaction (private communication). 
The total potential energy for $n$ particles located at $\mathbf{k}_{1}, \ldots, \mathbf{k}_{n}$ is then

$$
U\left(\mathbf{k}_{1}, \ldots, \mathbf{k}_{n}\right)=\sum_{1 \leqq i<j \leqq n} \Phi\left(\mathbf{k}_{j}-\mathbf{k}_{i}\right) .
$$

Let $K^{1}, \ldots, K^{v}$ be integers $>0$ and define a "box" $\Lambda(\mathbf{K})$ and its "volume" $V(\mathbf{K})$ by

$$
\begin{aligned}
& \Lambda(\mathbf{K})=\left\{\mathbf{k}: 0 \leqq k^{i}<K^{i} \text { for } i=1, \ldots, v\right\} \\
& V(\mathbf{K})=\prod_{i=1}^{v} K^{i} .
\end{aligned}
$$

The (configurational) canonical partition function is

$$
Q(\mathbf{K}, n)=\frac{1}{n !} \sum_{\mathbf{k}_{1} \in \Lambda(\mathbf{K})} \ldots \sum_{\mathbf{k}_{n} \in \Lambda(K)} e^{-\beta U\left(\mathbf{k}_{1}, \ldots, \mathbf{k}_{n}\right)}
$$

and the grand partition function at activity $\zeta$ is

$$
\boldsymbol{\Xi}(\mathbf{K}, \zeta)=\sum_{n} \zeta^{n} Q(\mathbf{K}, n) .
$$

Let us write $\mathbf{K} \rightarrow \infty$ if $K^{1} \rightarrow+\infty, \cdots, K^{v} \rightarrow+\infty$.

Proposition 1. 1. Let $K \rightarrow \infty, V(\mathbf{K})^{-1} n \rightarrow \varrho$ where $0<\varrho<1$; then the limit

$$
g(\varrho)=\lim V(\mathbf{K})^{-1} \log Q(\mathbf{K}, n)
$$

exists and is finite and concave in $\varrho$.

2. Let $\mathbf{K} \rightarrow \infty, \zeta>0$; then the limit

$$
P(\zeta)=\lim V(\mathbf{K})^{-1} \log \Xi(\mathbf{K}, \zeta)
$$

exists, is finite and satisfies

$$
P(\zeta)=\max _{\varrho}[\varrho \log \zeta+g(\varrho)] .
$$

A proof of Proposition 1, with the conditions (2.1) on the potential, does not seem to be published but is an easy exercise (published results on the thermodynamic limit include [8], [4], [3], [1]).

Furthermore if one assumes that $\Phi(\mathbf{k})$ vanishes except for a finite number of values of $k$, then Proposition 1 follows from [4].

Let us write

$$
C=\sum_{\mathbf{k} \neq 0} \Phi(\mathbf{k}) .
$$

Furthermore if $\Delta$ is a subset of $\Lambda(\mathbf{K})$, let $n(\Delta)$ be the number of elements in $\Delta$ and define

$$
\begin{gathered}
\hat{Q}(\mathbf{K}, n)=\sum_{n(\Delta)=n} \prod_{\mathbf{h}_{1} \in \Delta} \prod_{\mathbf{k}_{2} \in A(\mathbf{K})-\Delta} \exp \left(\frac{\mathbf{1}}{2} \beta \Phi\left(\mathbf{k}_{\mathbf{2}}-\mathbf{k}_{1}\right)\right) \\
\hat{\Xi}(\mathbf{K}, z)=\sum_{n} z^{n} \hat{Q}(\mathbf{K}, n) .
\end{gathered}
$$

Proposition 2. 1. If $\mathbf{K} \rightarrow \infty, V(\mathbf{K})^{-1} n \rightarrow \varrho$ where $0<\varrho<1$, then

$$
\lim V(\mathbf{K})^{-1} \log \hat{Q}(\mathbf{K}, n)=g(\varrho)+\frac{1}{2} \beta C \varrho=\hat{g}(\varrho) .
$$


2. If $\mathbf{K} \rightarrow \infty$ and $z=\exp \left(-\frac{1}{2} \beta C\right) \zeta>0$, then

$$
\begin{aligned}
\lim V(\mathbf{K})^{-1} \log \hat{\Xi}(K, z) & =\hat{P}(\zeta)=\hat{p}(z) \\
\hat{p}(z) & =\max _{\varrho}[\varrho \log z+\hat{g}(\varrho)] .
\end{aligned}
$$

Apart from boundary effects we would have $Q=\hat{Q} \cdot \exp \left(-\frac{1}{2} n \beta C\right)$ and $\boldsymbol{\Xi}=\hat{\boldsymbol{E}}$ (see [6]), but it is easily checked from (2.1) that the boundary effects disappear in the limit, proving Proposition 2.

Proposition 3. The following identities hold

$$
\hat{g}(\varrho)=\hat{g}(1-\varrho), \quad \hat{p}(z)=\log z+\hat{p}\left(z^{-1}\right) .
$$

Proposition 4. If $\Phi(\mathbf{k}) \leqq 0$ for $\mathbf{k} \neq 0$, then the polynomial $\hat{\Xi}(\mathbf{K}, z)$ in $z$ has its roots on the circle $|z|=1$. From this follows that $\hat{p}(z)$ is realanalytic on the intervals $[0,1)$ and $(1,+\infty)$ but may be non-analytic at $z=1$. The system may thus undergo at most one phase transition (for $z=1$ ).

Proposition 3 is obvious, Proposition 4 is a deep theorem by LeE and YANG [6].

According to Propositions 3 and 4 a first-order phase transition would be likely to occur as a horizontal segment in the graph of the function $\hat{g}$. To exhibit such a behaviour we shall make use of the following result.

Proposition 5. For each $\mathbf{K}$ we choose a set $\mathscr{P}_{\mathbf{K}}$ of subsets of $\Lambda(\mathbf{K})$ and define

If

$$
\begin{gathered}
Z(\Delta)=\prod_{\mathbf{k}_{1} \in \Delta} \prod_{\mathbf{k}_{2} \in \Lambda(\mathbf{K})-\Delta} \exp \left(\frac{1}{2} \beta \Phi\left(\mathbf{k}_{2}-\mathbf{k}_{1}\right)\right) \\
Z(\mathbf{K}, n)=\sum_{\Delta \in \mathscr{P}} \sum_{\mathbf{K}}, n(\Delta)=n \\
Z(\Delta) .
\end{gathered}
$$

$$
\lim _{\mathbf{K} \rightarrow \infty} V(\mathbf{K})^{-1} \log \sum_{n=0}^{V(K)} Z(\mathbf{K}, n)=\hat{p}(1)
$$

$$
\underset{\mathbf{K} \rightarrow \infty}{\lim . \inf }\left(\sum_{n=0}^{V(\mathbf{K})} Z(\mathbf{K}, n)\right)^{-1} \sum_{n=0}^{V(\mathbf{K})} V(\mathbf{K})^{-1} n Z(\mathbf{K}, n) \leqq \varrho_{0}<\frac{1}{2}
$$

then $\hat{g}$ reduces to a constant on the interval $\left[\varrho_{0}, 1-\varrho_{0}\right]$.

Given $\varepsilon>0$, there exists a sequence $\left(\mathbf{K}_{j}\right)$ such that

$$
\mathbf{K}_{j} \rightarrow \infty,\left(\sum_{n} Z\left(\mathbf{K}_{j}, n\right)\right)^{-1} \sum_{n} V\left(\mathbf{K}_{j}\right)^{-1} n Z\left(\mathbf{K}_{j}, n\right) \leqq \varrho_{0}+\varepsilon / 2 .
$$

Then,

$$
\left[\left(\sum_{n} Z\left(\mathbf{K}_{j}, n\right)\right)^{-1} \sum_{n \geqq\left(\varrho_{0}+\varepsilon\right) V\left(\mathbf{K}_{j}\right)} Z\left(\mathbf{K}_{j}, n\right)\right]\left(\varrho_{0}+\varepsilon\right) \leqq \varrho_{0}+\varepsilon / 2
$$


or

hence

$$
\left(\sum_{n} Z\left(\mathbf{K}_{j}, n\right)\right)^{-1} \sum_{n<\left(\varrho_{0}+\varepsilon\right) V\left(\mathbf{K}_{j}\right)} Z\left(\mathbf{K}_{j}, n\right) \geqq \frac{\varepsilon / 2}{\varrho_{0}+\varepsilon}
$$

$$
\lim _{j \rightarrow \infty} V\left(\mathbf{K}_{j}\right)^{-1} \log \sum_{n<\left(\varrho_{0}+\varepsilon\right) V\left(\mathbf{K}_{j}\right)} Z\left(\mathbf{K}_{j}, n\right)=\hat{p}(\mathbf{1}) .
$$

Let $n_{j}$ be such that

then

$$
Z\left(\mathbf{K}_{j}, n\right)=\max _{n<\left(\varrho_{0}+\varepsilon\right) V\left(\mathbf{K}_{j}\right)} Z\left(\mathbf{K}_{j}, n\right)
$$

$$
\lim _{j \rightarrow \infty} V\left(\mathbf{K}_{j}\right)^{-1} \log Z\left(\mathbf{K}_{j}, n_{j}\right)=\hat{p}(1) .
$$

Possibly taking a subsequence of $\left(\mathbf{K}_{j}\right)$ we may assume that

$$
V\left(\mathbf{K}_{j}\right) n_{j} \rightarrow \varrho_{1} \leqq \varrho_{0}+\varepsilon .
$$

Since $Z\left(\mathbf{K}_{j}, n_{j}\right) \leqq \hat{Q}\left(\mathbf{K}_{j}, n_{j}\right)$, it follows from (2.25) that

$$
\hat{p}(1) \leqq \hat{g}\left(\varrho_{1}\right) \leqq \hat{g}\left(\varrho_{0}+\varepsilon\right) \leqq \hat{g}\left(\frac{1}{2}\right)=\hat{p}(1)
$$

which proves Proposition 5.

\section{Existence of a first order phase transition}

Theorem. Consider a system of particles on the lattice of points $\mathbf{k}=\left(k^{1}, \ldots, k^{\nu}\right)$ with integral coordinates in $\nu$ dimensions, $v \geqq 2$. We assume that the particles interact through a pair potential $\Phi$ such that $\Phi(\mathbf{k})=\Phi(-\mathbf{k})$ and $\Phi(0)=+\infty$. Let $\Phi_{1}, \ldots, \Phi_{v}$ be the values of $\Phi$ for nearest neighbours in the directions of the $\nu$ coordinate axes and put

$$
D_{i}=\frac{1}{2} \sum_{\mathbf{k}}^{\prime}\left|k^{i}\right| \cdot|\Phi(\mathbf{k})|
$$

where $\Sigma^{\prime}$ extends over all $\mathbf{k}$ except 0 and the $2 v$ nearest neighbours of 0 .

If we have

$$
\Phi_{i}+D_{i}<0
$$

for $i=1, \ldots, v$, then the system undergoes a first order phase transition at low temperature.

Let us define

$$
\Lambda^{\prime}(\mathbf{K})=\left\{\mathbf{k} \in \Lambda(\mathbf{K}): 1 \leqq k^{i}<K^{i}-1 \text { for } i=1, \ldots, v\right\} .
$$

We shall base our proof of the above theorem on Proposition 5, taking for $\mathscr{P}_{\mathbf{K}}$ the set of subsets of $\Lambda^{\prime}(\mathbf{K})$, i.e. the set of configurations which have no point along the "boundary" of $\Lambda(\mathbf{K})$. Equation (2.18) is then clearly satisfied.

To evaluate the 1.h.s. of (2.19) we now follow DoBRusHIN and GRIFFITHs. Given $\Delta \in \mathscr{P}_{\mathbf{K}}$ we draw around each $\mathbf{k} \in \Delta$ the $2 v$ faces of the unit cube centered at $\mathbf{k}$ and suppress the faces which occur twice: we obtain 
in this way a closed polyhedron $\Gamma(\Delta)$. Each face of $\Gamma(\Delta)$ separates a point $\mathbf{k}_{\mathbf{1}} \in \Delta$ and a point $\mathbf{k}_{\mathbf{2}} \notin \Delta$. Along a $\boldsymbol{\nu}$-2-dimensional edge of $\Gamma(\Delta)$ there may be either 2 or 4 faces meeting. In the case of 4 faces, we deform slightly the polyhedron, "chopping off" the edge from the cubes containing a particle. When this is done $\Gamma(\Delta)$ splits into disconnected polyhedra $\gamma_{1}, \ldots, \gamma_{r}$, which we shall call cycles.

It will be convenient to consider a polyhedron $\Gamma(\Delta)$ as the set formed by the cycles into which it splits: $\Gamma(\Delta)=\left\{\gamma_{1}, \ldots, \gamma_{r}\right\}$. Given a cycle $\gamma$, we denote by $n(\gamma)$ the number of lattice points inside of $\gamma$ and by $|\gamma|_{i}$ the number of its faces orthogonal to the $i$-th coordinate axis. We call origin site of $\gamma$ the lattice point $\mathrm{k}$ inside of $\gamma$ which is smallest in lexicographic order.

We shall make use of the following easy lemmas which give in terms of the parameters $|\gamma|_{1}, \ldots,|\gamma|_{\nu}$ estimates of the entropy, number of particles, and energy associated with a cycle. It would be easy to obtain better estimates, which would improve the r.h.s. of (3.12) (see [2], [5]) but not our theorem.

Lemma 1. At least $v$ faces of the unit cube around the origin site of a cycle $\gamma$ belong to $\gamma$ (one orthogonal to each coordinate axis). Building up $\gamma$ face by face, with 3 possible orientations for each face, one finds that there are at most $\prod_{i=1}^{v} 3^{|\gamma|_{i}-1}$ cycles with $|\gamma|_{i}$ faces orthogonal to the $i$-th coordinate axis and given origin site, hence less than $V(\mathbf{K}) \prod_{i=1}^{v} 3^{|\gamma|_{i}-1}$ cycles with arbitrary origin site.

Lemma 2. If $\Gamma(\Delta)=\left\{\gamma_{1}, \ldots, \gamma_{r}\right\}$, then

and for any cycle $\gamma$

$$
n(\Delta) \leqq \sum_{j=1}^{r} n\left(\gamma_{j}\right)
$$

$$
n(\gamma) \leqq \prod_{i=1}^{v}\left(\frac{1}{2}|\gamma|_{i}\right)^{1 /(\nu-1)} .
$$

We leave the proofs of Lemma 1 (see [9]) and Lemma 2 to the reader.

Lemma 3. Let the cycle $\gamma$ belong to $\Gamma(\Delta)$ and let $\Delta^{\prime}$ be such that $\Gamma\left(\Delta^{\prime}\right)$ $=\Gamma(\Delta)-\{\gamma\} ;$ then

$$
Z(\Delta) \mid Z\left(\Delta^{\prime}\right) \leqq \exp \left[\frac{1}{2} \beta \sum_{i=1}^{\nu}|\gamma|_{i}\left(\Phi_{i}+D_{i}\right)\right] .
$$

To prove Lemma 3 notice that two lattice points which are both inside or outside of $\gamma$ yield the same contribution to $Z(\Delta)$ and $Z\left(\Delta^{\prime}\right)$ (see (2.16)). Each face of $\gamma$ separates two lattice points of which one is empty and the other occupied for $\Delta$, but both are empty or occupied for 
$\Delta^{\prime} ;$ this yields the factor $\exp \left[\frac{1}{2} \beta \sum_{i=1}^{\nu}|\gamma|_{i} \Phi_{i}\right]$ in (3.6). The number of ways in which $\mathbf{k}=\left(k^{1}, \ldots, k^{\nu}\right)$ may occur as the difference $\mathbf{k}_{1}-\mathbf{k}_{\mathbf{2}}$ or $\mathbf{k}_{2}-\mathbf{k}_{\mathbf{1}}$ with $\mathbf{k}_{1}$ inside of $\gamma$ and $\mathbf{k}_{2}$ outside of $\gamma$ is at most $\sum_{i=1}^{\nu}|\gamma|_{i}\left|k^{i}\right|$ (draw between $\mathrm{k}_{1}$ and $\mathrm{k}_{2}$ a broken line constituted of $v$ segments parallel to the coordinate axes; it must cross a face of $\gamma$ and if this face is orthogonal to the $i$-th coordinate axis, can do so in only $\left|k^{i}\right|$ ways). Therefore, apart from the factor due to nearest neighbours, $Z(\Delta)$ and $Z\left(\Delta^{\prime}\right)$ differ by at most a factor

$$
\exp \left[\frac{1}{4} \beta \sum_{\mathrm{k}}^{\prime}|\Phi(\mathbf{k})| \sum_{i=1}^{v}|\gamma|_{i}\left|k^{i}\right|\right]=\exp \left[\frac{1}{2} \beta \sum_{i=1}^{v}|\gamma|_{i} D_{i}\right],
$$

which concludes the proof.

We come now to the proof of the theorem. We notice that by (3.4)

$$
\begin{aligned}
\sum_{\Delta \in \mathscr{P}_{\mathbf{K}}} V(\mathbf{K})^{-1} n(\Delta) Z(\Delta) & \leqq \sum_{\Delta \in \mathscr{O}_{\mathbf{K}}} Z(\Delta) \sum_{j=1}^{r} V(\mathbf{K})^{-1} n\left(\gamma_{j}\right) \\
& =\sum_{\gamma} V(\mathbf{K})^{-1} n(\gamma) N(\gamma),
\end{aligned}
$$

where

By Lemma 3 we have

$$
N(\gamma)=\sum_{\Gamma(\Delta) \ni \gamma} Z(\Delta)
$$

$$
\begin{aligned}
\left(\sum_{\Delta \in \mathscr{O}_{\mathbf{K}}} Z(\Delta)\right)^{-1} N(\gamma) & \leqq \sum_{\Gamma(\Delta) \ni \gamma} Z(\Delta) \mid \sum_{\Gamma(\Delta) \ni \gamma} Z\left(\Delta^{\prime}\right) \leqq \\
& \leqq \exp \left[\frac{1}{2} \beta \sum_{i=1}^{\nu}|\gamma|_{i}\left(\Phi_{i}+D\right)\right] .
\end{aligned}
$$

By (3.8), (3.10) and (3.5)

$$
\begin{gathered}
\left(\sum_{\Delta \in \mathscr{P}_{\mathbf{K}}} Z(\Delta)\right)^{-1} \sum_{\Delta \in \mathscr{P}_{\mathbf{K}}} V(\mathbf{K})^{-1} n(\Delta) Z(\Delta) \leqq \\
\leqq \sum_{\gamma} V(\mathbf{K})^{-1} \prod_{i=1}^{v}\left(\frac{1}{2}|\gamma|_{i}\right)^{1 /(\nu-1)} \exp \left[\frac{1}{2} \beta \sum_{i=1}^{v}|\gamma|_{i}\left(\Phi_{i}+D\right)\right] .
\end{gathered}
$$

Replacing the sum over $\gamma$ by a sum over $|\gamma|_{1}=2 l_{1}, \ldots,|\gamma|_{v}=2 l_{v}$ we obtain thus by Lemma 1

$$
\begin{gathered}
\left(\sum_{\Delta \in \mathscr{P}_{\mathbf{K}}} Z(\Delta)\right)^{-1} \sum_{\Delta \in \mathscr{P}_{\mathbf{K}}} V(\mathbf{K})^{-1} n(\Delta) Z(\Delta) \leqq \\
\leqq \\
\sum_{l_{1}=1}^{\infty} \ldots \sum_{l_{\nu}=1}^{\infty} \prod_{i=1}^{\nu}\left(l_{i}^{1 /(\nu-1)} 3^{2 l_{i}-1} \exp \left[l_{i} \beta\left(\Phi_{i}+D\right)\right]\right) \\
=\prod_{i=1}^{v}\left[\sum_{l=1}^{\infty} l^{1(\nu-1)} 3^{2 l-1} \exp \left(l \beta\left(\Phi_{i}+D\right)\right)\right] .
\end{gathered}
$$


It is immediate that if (3.2) holds then, for sufficiently large $\beta$, the r.h.s. of (3.12) is smaller than $\frac{1}{2}$ and the theorem follows from Proposition 5.

Remark. The result we have obtained could of course be easily extended to more general lattices. Furthermore, by well-known arguments, it is possible to translate it into statements about spin systems in a magnetic field or about mixtures. This yields in particular a proof of the spontaneous magnetization of a ferromagnet under fairly realistic assumptions. On the other hand the method could be applied for instance to prove the segregation into two phases of a mixture of two different kinds of dimers on a lattice in the close-packing limit.

Acknowledgements. One of us (A. G.) wishes to thank Monsieur L. Motchane for his kind hospitality at the I.H.E.S.

\section{References}

[1] Dobrushin, R. L.: Teorija verojatn. i ee prim. 9, 626 (1964).

[2] - Teorija verojatn. i ee prim. 10, 209 (1965).

[3] Fisher, M. E.: Arch. Rat. Mech. Anal. 17, 377 (1964).

[4] Griffiths, R.: J. Math. Phys. 5, 1215 (1964).

[5] - Phys. Rev. 136, A 437 (1964).

[6] Lee, T. D., and C. N. YANG: Phys. Rev. 87, 410 (1952).

[7] Peierls, R.: Proc. Cambridge Phil. Soc. 32, 477 (1936).

[8] Ruelle, D.: Helv. Phys. Acta 36, 183 (1963).

[9] van Der Waerden, B. L.: Z. Physik 118, 473 (1941).

[10] YANG, C. N.: Phys. Rev. 85, 808 (1952). 